\title{
Efeitos da estimulaçáo elétrica funcional no controle neuromuscular artificial
}

\author{
Effects of functional electrical stimulation in artificial neuromuscular control
}

\author{
Eddy Krueger-Beck ${ }^{1}$,Eduardo Mendonça Scheeren ${ }^{2}$, Guilherme Nunes \\ Nogueira Neto ${ }^{3}$, Vera Lúcia da Silveira Nantes Button ${ }^{4}$, Percy Nohama ${ }^{5}$
}

\begin{abstract}
RESUMO
Regióes distintas do sistema nervoso central ativam o sistema neuromuscular. Atualmente, utilizam-se sistemas artificiais para mimetizarem as açóes fisiológicas perdidas devido a uma lesão neurológica. A estimulação elétrica de tecidos humanos in vivo, como forma de tratamento, desenvolveu-se por meio de pesquisas e do aprimoramento da tecnologia. Pessoas que sofreram lesão medular podem perder parcial ou totalmente a funçáo motora de uma determinada região corporal. A aplicaçáo de estimulação elétrica no tecido neuromuscular gera movimentaçáo artificial que pode desenvolver uma melhora de longo prazo através da plasticidade neuronal. A estimulaçáo elétrica funcional pode utilizar variados parâmetros, eletrodos e locais de aplicação. Quando o ajuste e correção dos parâmetros estimulatórios ocorre manualmente, tem-se um sistema em malha aberta, quando ocorre automaticamente, o sistema denomina-se controle em malha fechada. Ambas as formas contribuem para a reabilitação física de pacientes acometidos por lesão neuronal. Os sistemas em malha fechada apresentam vantagens em relação aos de malha aberta, como a correção automática dos parâmetros de estimulação. Assim, com o desenvolvimento de estratégias de controle e a criação de interfaces amigáveis, a ativaçáo do conjunto de equipamentos e softwares que viabilizarão o movimento artificial poderá ser efetuada pelo próprio usuário, assemelhando-se ao sistema fisiológico humano.
\end{abstract}

Unitermos. Estimulação Elétrica, Medula Espinhal, Plasticidade Neuronal, Reabilitaçáo.

Citaçáo. Krueger-Beck E, Scheeren EM, Nogueira Neto GN, Button VLSN, Nohama P. Efeitos da estimulação elétrica funcional no controle neuromuscular artificial.

Trabalho realizado no Laboratório de Engenharia da Reabilitaçáo (LER) da Universidade Tecnológica Federal do Paraná (UTFPR), Campus Curitiba, Curitiba-PR, Brasil.

1. Fisioterapeuta, Doutorando em Engenharia Biomédica pela UTFPR, Bolsista CAPES. Curitiba-PR, Brasil.

2. Educador Físico, MSc. em Ciências do Movimento Humano pela UFRGS RS, Doutorando em Engenharia Biomédica pela UTFPR, Curitiba-PR, Brasil. Bolsista CAPES.

3. Engenheiro de Computação, Doutorando em Engenharia Biomédica pela UNICAMP, Bolsista CNPQ. Campinas-SP, Brasil.

4. Doutora em Engenharia Biomédica pela UNICAMP, Campinas-SP, Docente da UNICAMP, Campinas-SP, Brasil.

5. Doutor em Engenharia Biomédica pela UNICAMP, Campinas-SP, Docente da PUCPR e da UTFPR, Curitiba-PR, Brasil.

\begin{abstract}
Different regions of the central nervous system activate the neuromuscular system. Nowadays, artificial systems are employed to imitate physiological tasks lost due to neurological injuries. The electrical stimulation of in vivo human tissues, as a way of treatment, has been developed by means of research and technological enhancements. People who have suffered spinal cord injury can lose partial or total motor function. The electrical stimulation of neuromuscular tissue generates artificial movements that can afford long term health improvements by means of neuronal plasticity. Functional electrical stimulation can use diverse parameters, electrodes and application sites in the body. When the adjustments and corrections of stimulatory parameters are performed manually, the system is called open loop control; whereas when it is automatic, the system is closed-loop control. Both ways (open- and closed-loop) contribute to physical rehabilitation of spinal cord injury patients. Closed-loop control systems present advantages in comparison to open-loop systems like automatic correction of electrical stimulation parameters. Therefore, with the development of artificial motor control strategies and the creation of user-friendly interfaces, the activation of software and hardware for producing movements artificially can be performed by the users of the system (spinal cord injury patients), resembling the human physiological system.
\end{abstract}

Keywords. Electric Stimulation, Spinal Cord, Neuronal Plasticity, Rehabilitation.

Citation. Krueger-Beck E, Scheeren EM, Nogueira Neto GN, Button VLSN, Nohama P. Effects of functional electrical stimulation in artificial neuromuscular control.
Endereço para correspondência: Eddy Krueger-Beck Universidade Tecnológica Federal do Paraná (UTFPR) Laboratório de Engenharia de Reabilitação - CPGEI Av. Sete de Setembro 3165, Rebouças CEP 80230-901, Curitiba-PR, Brasil.

E-mail: kruegereddy@gmail.com/ escheeren@gmail.com/guinnn@gmail.com/ vera@ceb.unicamp.br/ percy.nohama@gmail.com 


\section{INTRODUÇÃO}

Relatos históricos afirmam que no ano 46 a.C. Scribonius Largus utilizava-se de peixes torpedo, que geravam tensóes elétricas entre 25 a $30 \mathrm{~V}$, para o tratamento de cefaléias e dores decorrentes de artrite gotosa ${ }^{1,2}$. Em 1791, Luigi Galvani publicou seus comentários a respeito dos efeitos da eletricidade sobre o movimento muscular ${ }^{3}$, importante obra onde descreveu estudos de estimulação elétrica nas patas traseiras de râs com a carga estática proveniente de duas superfícies metálicas distintas ${ }^{1,2,4}$. Em 1855, o médico francês Guillaume Duchenne de Boulogne publicou um trabalho descrevendo o uso de estimulação elétrica (EE) sobre tecidos musculares, além de ser o primeiro pesquisador a ter sucesso na estimulação do nervo frênico5.

Atualmente, a abrangência de aplicação da EE vai além do foco desta revisão que está voltada para o controle motor. Na literatura, estudos descrevem a aplicação de EE por meio de eletrodos implantáveis em órgãos sensoriais como retina e cóclea ${ }^{2}$ e trabalhos com estimulação cerebral profunda para distúrbios neurológicos como mal de Parkinson, síndrome de Tourette, epilepsia e transtorno obsessivo compulsivo ${ }^{1,6,7}$. Ainda são encontradas pesquisas envolvendo EE aplicada à acupuntura ${ }^{8}$, à regeneração tecidual como a do tecido nervoso ${ }^{9}$, à analgesia por aplicação na região cerebral profunda, no córtex motor ou na medula espinal ${ }^{1,6,10}$.

A utilização de EE no tecido contrátil está associada a diversas aplicaçóes como: (1) profilaxia de contraturas e trombose venosa profunda ${ }^{11}$, (2) redução de padrão motor espástico, bipedestação e deambulaçãoo ${ }^{1,2,12}$, (3) melhora da condição cardiopulmonar ${ }^{13,14}$ e metabólica ${ }^{15}$, (4) controle de movimentos de membro superior ${ }^{16}$ como a preensão manual ${ }^{2,10,17,18}$, (5) movimentos para vencer a ação da gravidade ${ }^{19}$ e movimentos finos como escrever ${ }^{20}$, (6) respiração artificial com estimulação do nervo frêni$\mathrm{co}^{5}$, (7) marca-passo cardíaco e (8) esvaziamento da bexiga e fortalecimento do assoalho pélvico ${ }^{1,2,7}$.

A paraplegia é uma condição neurológica proveniente de lesão medular (LM) ao nível torácico, lombar ou sacral, acarretando perda motora e/ou sensorial ${ }^{21}$. Os principais fatores que contribuem para o aumento das estatísticas no número de pessoas que sofreram LM são quedas, acidentes esportivos e de trânsito, sendo este o que possui maior incidência entre os jovens ${ }^{22,23}$. O processo de recuperação de pacientes com paraplegia requer diversos cuidados como a utilização de órteses para posicionar o joelho em extensão, o treino de bipedestação e deambulação, o controle e tratamento de possíveis alteraçóes motoras existentes (como hipotonia, espasticidade e hipertonia) e a higienização quando não apresentam controle vesical e intestinal ${ }^{13}$.

Dois tipos de nomenclatura são empregados na literatura para descrever a utilização de EE no neurônio motor em função das características do paciente. Quando a EE é aplicada em pessoas com LM objetivando produzir uma função corpórea é denominada estimulação elétrica funcional (FES - acrônimo do inglês functional electrical stimulation $)^{2,24}$ ou como estimulação neuromuscular funcional (do inglês functional neuromuscular stimulation) ${ }^{25}$. Já quando o objetivo da EE é gerar movimentos em pacientes hígidos, alguns autores utilizam a nomenclatura estimulação neuromuscular ${ }^{26}$ (NMES - do inglês neuromuscular electrical stimulation $\left.{ }^{27}\right)$. Doravante, como ainda não há um consenso da literatura em relação à nomenclatura em português, o termo FES será empregado para descrever EE visando à realização de movimento funcional, tanto em voluntários hígidos quanto em portadores de LM.

\section{MÉTODO}

Neste artigo, apresenta-se sucintamente o estado atual da ciência e da tecnologia sobre controle neuromuscular artificial com o uso da FES, descrevendo a estrutura envolvida para o controle, suas principais aplicaçóes, parâmetros estimulatórios, local de aplicação e tarefas realizadas. A pesquisa bibliográfica foi realizada nas bases Springer, ScienceDirect, Pubmed, Google Acadêmico e pelo portal de periódicos da CAPES. O idioma de preferência selecionado foi o inglês, com as keywords: functional electrical stimulation (FES), neuronal plasticity, locomotor activity, rehabilitation, fatigue e spinal cord injury (SCI). Realizou-se a procura de artigos com ampla janela de tempo, de 1960 a 2010, tanto no tema de estimulaçáo elétrica quanto em sistemas de controle motor. Também foram consultados livros considerados referências na área de reabilitação com utilização de FES. 


\section{Estado da arte}

Comercialmente, além da FES, os tipos de estimulação elétrica para gerar contração muscular são a corrente russa (de Kots) ${ }^{28}$ e a interferencial (heteródina) ${ }^{24,26} \mathrm{com}$ objetivo de fortalecimento. Quando a FES é utilizada concomitantemente à órtese, com o intuito de auxiliar o usuário a manter a posição ortostática ou deambular, a nomenclatura passa a ser sistema de FES híbrida ${ }^{29,30}$. A utilizaçáo da FES em musculatura com perda de função motora com o objetivo de criar movimento artificial, denomina-se prótese neural ${ }^{2}$.

A alimentaçãa do estimulador elétrico pode vir diretamente da rede elétrica ou por meio de baterias, o que é mais aplicado aos aparelhos portáteis ${ }^{12,31}$. Os parâmetros elétricos controlados na FES são a duração dos pulsos $(\mu s)$, a amplitude $(\mathrm{V})$, a intensidade (A) e a frequência dos pulsos $(\mathrm{Hz})$. Quando a modulação da FES ocorre variando-se a amplitude, considera-se a estimulação como modulada por amplitude de pulso (do inglês, pulse amplitude modulation - PAM), ao passo que quando controlada pela duração do pulso, a estimulação é classificada como modulada por largura de pulsos (do inglês, pulse width modulation - PWM ${ }^{32}$. A duração do pulso é denominada período ativo (on) e ao intervalo entre pulsos denomina-se período inativo (off). Geralmente, a frequência da portadora chega a milhares de hertz. Formatan- do a envoltória da fre-quência portadora, tem-se o burst, que compreende uma sequência de pulsos, denominada trem de pulsos, e cuja duração define o período ativo do burst. Contudo, o período inativo do burst, caracterizado pela ausência de pulsos, possui um tempo de duração mais prolongado ${ }^{28}$ (Figura 1). Os pulsos podem ser bifásicos (simétricos ou assimétricos) ou monofásicos ${ }^{2,10}$, com diferentes formas de onda como retangular, serrilhada e senoidal (normal ou modulada) ${ }^{33}$. Frequências inferiores a $20 \mathrm{~Hz}$ provocam contração fasciculada (contração de poucos grupos de fascículos do tecido muscular desenvolvendo pouca resposta de força), que é ineficaz para geração de movimentos funcionais e superiores a $70 \mathrm{~Hz}^{24,26}$ provocam desconforto sensorial em pacientes com sensibilidade nociceptiva presente ${ }^{16,34,35}$.

Uma condição importante para a aplicação de FES é o grau de inervação e hipotrofia muscular. Quanto maior o nível de desnervação e hipotrofia, maior é a corrente necessária para despolarizar as células nervosas e musculares, o que pode inviabilizar o tratamento em certos casos ${ }^{10}$. Uma técnica alternativa à aplicação direta de eletricidade por meio de eletrodos no corpo humano é a estimulação nervosa por meio de campos magnéticos alternados. Esses campos geram uma diferença de potencial na membrana da célula nervosa capaz de despolarizar e causar a propagação de um potencial de ação. Outras

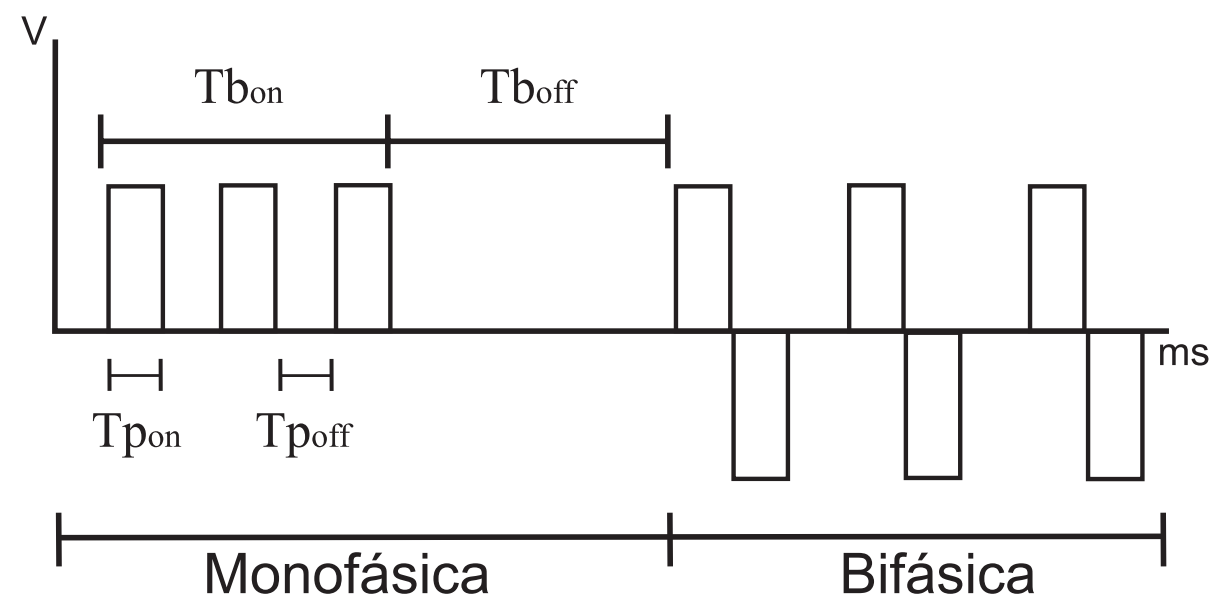

$\mathrm{Tb}_{\mathrm{on}}$ - período ativo do burst; $\mathrm{Tb}_{\mathrm{off}}$ - período inativo do burst; $\mathrm{T} \mathrm{p}_{\mathrm{on}}$ - período ativo do pulso; $\mathrm{T} \mathrm{p}_{\text {off }}$ - período de repouso do pulso.

Figura 1. Duração de pulso com onda quadrada e de burst. Tempos de alta ( $\mathrm{T} \mathrm{p}_{\text {on }}$ ) e de baixa do pulso ( $\mathrm{T} \mathrm{p}_{\text {off }}$ ) (em $\mu$ s) informam a frequência da portadora. On e off do burst (em ms) informam a duração do burst. Monofásica - pulso em apenas uma polaridade (positiva ou negativa); Bifásica - pulso alterna a sua polaridade a cada pulso. 
tecnologias como a estimulação magnética transcraniana ou a estimulação com laser de infravermelho de curta duração ${ }^{6}$ possuem o objetivo de estimular tecidos, assim como a FES.

Metodologicamente, a FES pode ser aplicada por meio de eletrodos transcutâneos (ou de superfície), percutâneos (posicionados abaixo da pele por meio de punção, os cabos estendem-se para o meio exterior e são conectados aos módulos externos) ou implantados (procedimento cirúrgico). Os eletrodos de superfície são colocados sobre a pele e podem ser auto-adesivos ou de silicone-carbono com gel ${ }^{24}$. Quando se tem um par de eletrodos, um ativo e outro de referência, a FES é uni- ou monopolar. Quando a FES possui dois eletrodos ativos e mais um eletrodo como referência, a aplicação é bipo$\operatorname{lar}^{32}$. Eletrodos de mesmo tamanho, por exemplo, os dois eletrodos com medida $5 \mathrm{~cm} \times 5 \mathrm{~cm}$, definem a estimulaçáo elétrica como bipolar ${ }^{36}$. Eletrodos com tamanhos distintos, por exemplo, um com medida $5 \mathrm{~cm}$ x $5 \mathrm{~cm}$ e outro com $5 \mathrm{~cm} \times 9 \mathrm{~cm}$, atribui-se a essa aplicação como monopolar, sendo que o menor eletrodo $(5 \mathrm{~cm} \times 5 \mathrm{~cm})$ terá uma maior densidade de corrente.

A impedância tecidual tem grande variação dependendo do acoplamento do eletrodo. Com uma frequência de $60 \mathrm{~Hz}$, a pele seca tem uma impedância aproximada de $93,0 \mathrm{k} \Omega / \mathrm{cm}^{2}$; já eletrodos (silicone-carbono) com gel sobre a pele reduzem a impedância para $10,8 \mathrm{k} \Omega / \mathrm{cm}^{2}$; eletrodos implantáveis apresentam $0,2 \mathrm{k} \Omega / \mathrm{cm}^{2} 37$ e podem ser posicionados ao redor do tecido nervoso ou no meio intramuscular ${ }^{2,10}$; o que melhora significativamente os efeitos da FES. Em relação ao desempenho da marcha em pacientes com LM, eletrodos transcutâneos são menos seletivos, necessitando de maior amplitude do estimulador elétrico, quando comparados aos implantáveis ${ }^{38}$. Isso se deve à necessidade da utilização de vários eletrodos e, especialmente, pela dificuldade de se atingir músculos profundos. Os eletrodos implantáveis devem ser de material biocompatível para evitar processos inflamatórios. Esses processos ocasionam alteração da impedância dos tecidos adjacentes (tegumentar, conjuntivo e muscular), dificultando a aplicação da $\mathrm{FES}^{39}$.

A literatura descreve diferentes posicionamentos de eletrodos de FES com o objetivo de maximizar a densidade de corrente, o que contribui para uma EE mais efetiva. Utilizou-se um eletrodo com $140 \mathrm{~cm}^{2}$ (ânodo) sobre o ventre do músculo gastrocnêmio e outro eletrodo com $3,14 \mathrm{~cm}^{2}$ (cátodo) sobre o ponto motor do músculo tibial anterior ${ }^{40}$, obtendo uma densidade de corrente 45 vezes maior no cátodo. Por meio de biópsia em tecido muscular de portadores de LM, evidenciou-se aumento enzimático devido ao treinamento realizado com a FES ${ }^{41}$.

O uso de FES também influencia as condiçôes cardiopulmonar e metabólica, como na captação da glicose sanguínea e oxidação do carboidrato ${ }^{15}$. Foi desenvolvido um sistema de FES aplicada à musculatura diafragmática com acionamento por sensor de temperatura, sincronizando a ativação da FES com o período inspiratório ${ }^{42}$. Foram evidenciados esses benefícios realizando um experimento com aplicação de FES visando a melhora da condição cardiopulmonar em pacientes hígidos ${ }^{14}$. O protocolo experimental consistiu da aplicação de FES sobre o nervo fibular comum durante um período de $2 \mathrm{~min}$ com intensidade suficiente para gerar $50 \%$ da contração voluntária máxima com frequência de burst de $25 \mathrm{~Hz}$. Na comparação dos resultados experimentais com o grupo controle, no qual se realizou somente contração voluntária, constatou-se que o grupo experimental foi o que obteve maior nível de saturação de $\mathrm{O}_{2} 1 \mathrm{~min}$ após o término do exercício, $119,7 \% \pm 8,9 \%$ contra $104,8 \pm 5,7 \%$ do grupo controle.

O sistema nervoso central ( $\mathrm{SNC}$ ) possui vias de estímulos eferentes ${ }^{43,44}$ que são responsáveis pela ativação muscular, glandular, dentre outras funçóes. O SNC também possui uma via de estímulos aferentes ${ }^{43-45}$ e que oferece realimentação ${ }^{46}$ de uma série de informações, tais como localização espacial do segmento, velocidade de deslocamento e sensibilidades tátil e nociceptiva ${ }^{47}$. As vias neuronais proprioceptivas são de fundamental importância para a deambulação humana ${ }^{48}$, pois enviam estímulos aferentes que corrigem os desequilíbrios que ocorrem durante a marcha ${ }^{49}$, contribuindo com o sistema de servo-controle da medula espinal ${ }^{50}$. Uma proposta didática à explicação do mecanismo de controle motor compara a uma marionete, como mostra a Figura 2, onde o ventríloquo representaria o SNC, os cordóes as vias neuronais motoras e aferentes e o boneco o sistema músculo-esquelético ${ }^{51}$.

FES traz benefícios a portadores de alteração de movimento, como a paralisia decorrente da $\mathrm{LM}^{52}$. Além 


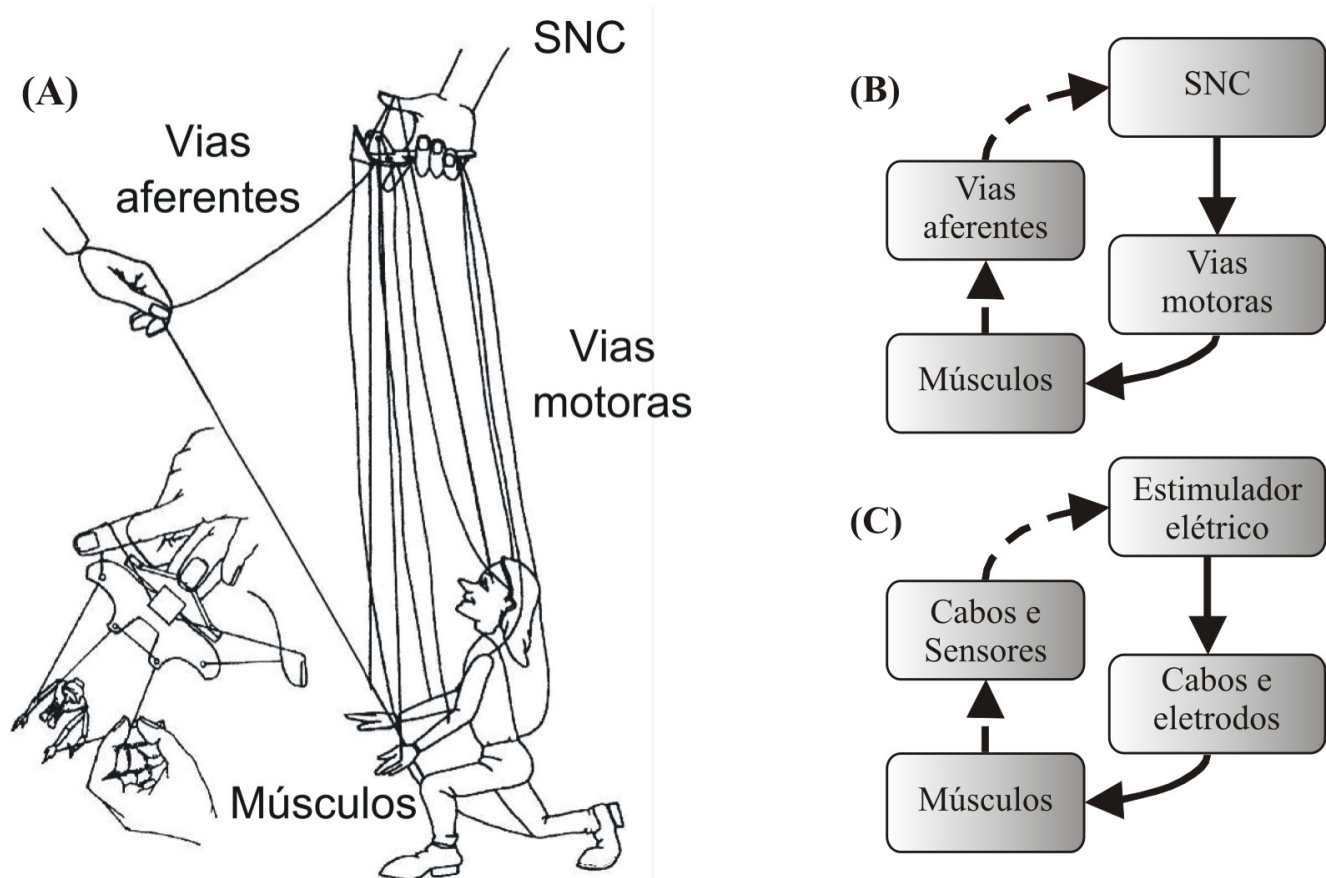

SNC - Sistema nervoso central

Figura 2. Representação modificada do controle motor segundo Loeb ${ }^{51}$. Em (A), sistema de controle motor coordenado pelo SNC com informações de saída e entrada, em (B) seu diagrama em blocos e em (C) diagrama em blocos do controle neuromuscular artificial.

das vantagens da capacidade de locomoção com a aplicação de FES, constata-se que seu uso acarreta em ganho de massa muscular e estimula a remodelagem neuronal ${ }^{43,47,53}$ reorganizando as vias neuronais ${ }^{54,55}$. Essa plasticidade de longo prazo é representada por (1) decremento da ativação da musculatura antagonista, (2) aumento da atividade da unidade motora e (3) incremento na ativação da musculatura sinergista ${ }^{56}$. A remodelagem neuronal ocorre devido à estimulação local sobre o músculo ou ativação antidrômica (sentido terminal do axônio para o soma) ${ }^{57}$ nas células motoras do corno anterior da medula espinal. Sinapses de Hebb, ocorrem em regióes do SNC que estão associadas à memória e ao aprendizado, tanto sensorial quanto motor, utilizando-se do potencial de ação de longa duração presente, principalmente, nos neurônios que utilizam Glutamato como neurotransmissor nos receptores NMDA e AMPA. Sinapses desse tipo estão presentes ao nível das células do corno anterior da medula espinal ${ }^{58}$. A eficácia de uma abordagem reabilitacional depende do uso de FES associado a algum sistema de realimentação provido pelo paciente, onde o papel da FES é executar a função do movimento perdido e o paciente deve in- terpretar o movimento como se estivesse sendo realizado voluntariamente. Essa estratégia permite uma ativação de vias neuronais que, consequentemente, favorece uma remodelagem neuronal ${ }^{58}$.

O controle motor por meio de FES pode ser realizado de duas formas, as quais estão relacionadas ao conceito de controle em malha fechada e malha aberta. Entende-se por malha aberta um sistema de controle que não possui realimentação automatizada. Esse sistema consiste em aplicar um sinal (estímulo elétrico) na entrada de uma planta (músculo ou nervo motor), esperando-se que a variável controlada na saída consiga atingir um determinado valor ou apresente o comportamento desejado (movimento planejado). Nos sistemas em malha aberta e fechada existe um módulo chamado controlador, cujo sinal de entrada depende da saída na configuração em malha fechada. Em malha aberta, a entrada não depende da saída. Já em malha fechada, as informaçóes monitoradas na saída (por meio de sistemas de medição) realimentam a entrada do controlador. A partir de tais informaçóes, o sistema de controle define o nível de ajuste a ser aplicado ao estímulo, corrigindo eventuais erros e buscando atingir o objetivo desejado ${ }^{25}$. 
Como descrito anteriormente, em um sistema de malha aberta ${ }^{59}$, o permanente ajuste dos parâmetros eletrofisiológicos e/ou biomecânicos, tais como a adaptação ${ }^{60}$ nervosa frente à estimulação elétrica ${ }^{61}$ ou fadiga ${ }^{24}$, são corrigidos manualmente pelo terapeuta ou operador que reajusta os parâmetros elétricos do estimulador elétrico. Já em malha fechada ${ }^{62,63}$, sistemas de medição para aquisição de sinais biomecânicos ou eletrofisiológicos são utilizados no sistema de realimentação do estimulador elétrico. A utilização de estratégias de controle tanto em malha aberta quanto em malha fechada requerem o uso de órteses de membros inferiores (MMII) ou dos membros superiores (MMSS) para treino de bipedestação el ou deambulação em paraplégicos; entretanto, o sistema em malha fechada tende a ter uma resposta melhor ${ }^{64}$. A vantagem da utilização de sistemas em malha fechada é que eles mantêm o nível de angulação e/ou torque, por meio do incremento da intensidade estimulatória da FES ao longo do tempo e buscam retardar a perda de torque articular decorrente da fadiga muscular ${ }^{40}$. Também proporcionam a correção de possíveis variaçôes musculares decorrentes da espasticidade ${ }^{65}$. Os principais sistemas de medição utilizados em malha fechada englobam acelerômetros, giroscópios, microfones, dispositivos de laser, dinamômetros, plataformas de força, strain-ganges, eletrogoniômetros, eletromiógrafos (EMG) e células de carga ${ }^{2,62,66-69}$. A técnica de medição denominada mecanomiografia $(M M G)^{70}$ ou vibromiografia ${ }^{71}$, mede a oscilaçáo proporcionada pelo tecido muscular durante a contração ${ }^{72}$. A MMG pode ser utilizada em conjunto com a $\mathrm{FES}$, por não sofrer interferência elétrica $\operatorname{diret}^{73}$. Os sistemas de medição mecanomiográfica podem ser construídas com microfones ou sensores laser para medição de distância que fornecem um sinal monoaxial ${ }^{74}$. Foi utilizado MMG de laser para avaliar a contração muscular eliciada por FES no músculo bíceps braquial de pacientes hígidos e mostraram que, variando-se a duração do pulso, obtém-se variação similar no sinal de $\mathrm{MMG}^{69}$. A MMG é utilizada em conjunto com a FES para avaliar a resposta muscular durante a fadiga muscular e/ou adaptação do motoneurônio ${ }^{75}$ e para encontrar parâmetros ótimos para a $\mathrm{FES}^{76}$. O acelerômetro é um dispositivo que também pode ser utilizado na medição de MMG e pode fornecer sinais de vibração em um, dois ou três eixos ${ }^{72}$.
Softwares de controle são utilizados para melhorar a atividade da FES, garantindo maior estabilidade em malha fechada por meio da modelagem matemática. A literatura apresenta estratégias de controle utilizando técnicas, como redes neurais ${ }^{77}$ (contribuindo para sustentação do torque muscular ${ }^{78-80}$ ) ou lógica fuzzy para movimentação articular com a FES ${ }^{81}$. Sistemas mais avançados, além da realimentação da FES, permitem a antecipação (do inglês - feedforward $)^{82}$ do sistema músculo-esquelético para elaboração do movimento subsequente ${ }^{80}$. Antecedendo a FES, pode-se realizar uma simulação computacional do movimento modelando um sistema híbrido por meio de órtese recíproca THKAFO (tronco, quadril, joelho, tornozelo e pé) com controle em malha fechada ${ }^{30}$. Estudos recentes mostram que a ativação da FES para próteses neurais pode ser realizada pelo córtex motor do próprio paciente, isto é, com o controle proveniente do próprio usuário $^{46,83}$, definindo um sistema híbrido para um sistema de controle de FES por interface cérebro-máquina ${ }^{84}$.

A Tabela 1 apresenta uma sinopse cronológica de pesquisas que utilizaram FES para evocação de diferentes movimentos funcionais, além da apresentação dos parâmetros, sistemas de medição, voluntário (com LM ou hígido) e o objetivo das tarefas realizadas.

No começo da década de 1960, Kantrowitz utilizou FES transcutânea e demonstrou a possibilidade de um paciente paraplégico atingir a posição ortostática com o auxílio de muletas ${ }^{62}$. Para a realização do movimento, os músculos quadríceps femoral e glúteo máximo foram estimulados. O estudo evidenciou a necessidade de desenvolver um sistema de segurança para a ação de sentar e a necessidade de realizar um treino prévio para evitar a ocorrência de fadiga muscular ${ }^{2}$, fato que contribuiu para a melhor adequação da estrutura laboratorial para os estudos subsequentes. Na mesma década, Liberson et al. utilizaram FES no tratamento de pé equino com estimulação do nervo fibular comum, produzindo dorsiflexão e eversão do pé durante a fase de balanço da marcha ${ }^{10,85}$.

Já em 1987, relatavam-se movimentos mais elaborados realizados por meio de FES percutânea em paraplégicos. Os movimentos eliciados artificialmente objetivaram subir escadas e deambular com órteses. Foram estimulados os músculos responsáveis pela flexão, extensão, abdução e adução da pelve, os responsáveis pela extensão do joelho e 
Tabela 1

Parâmetros para controle neuromuscular artificial e atividades realizadas, encontradas em pesquisas entre os anos de 1987 a 2010

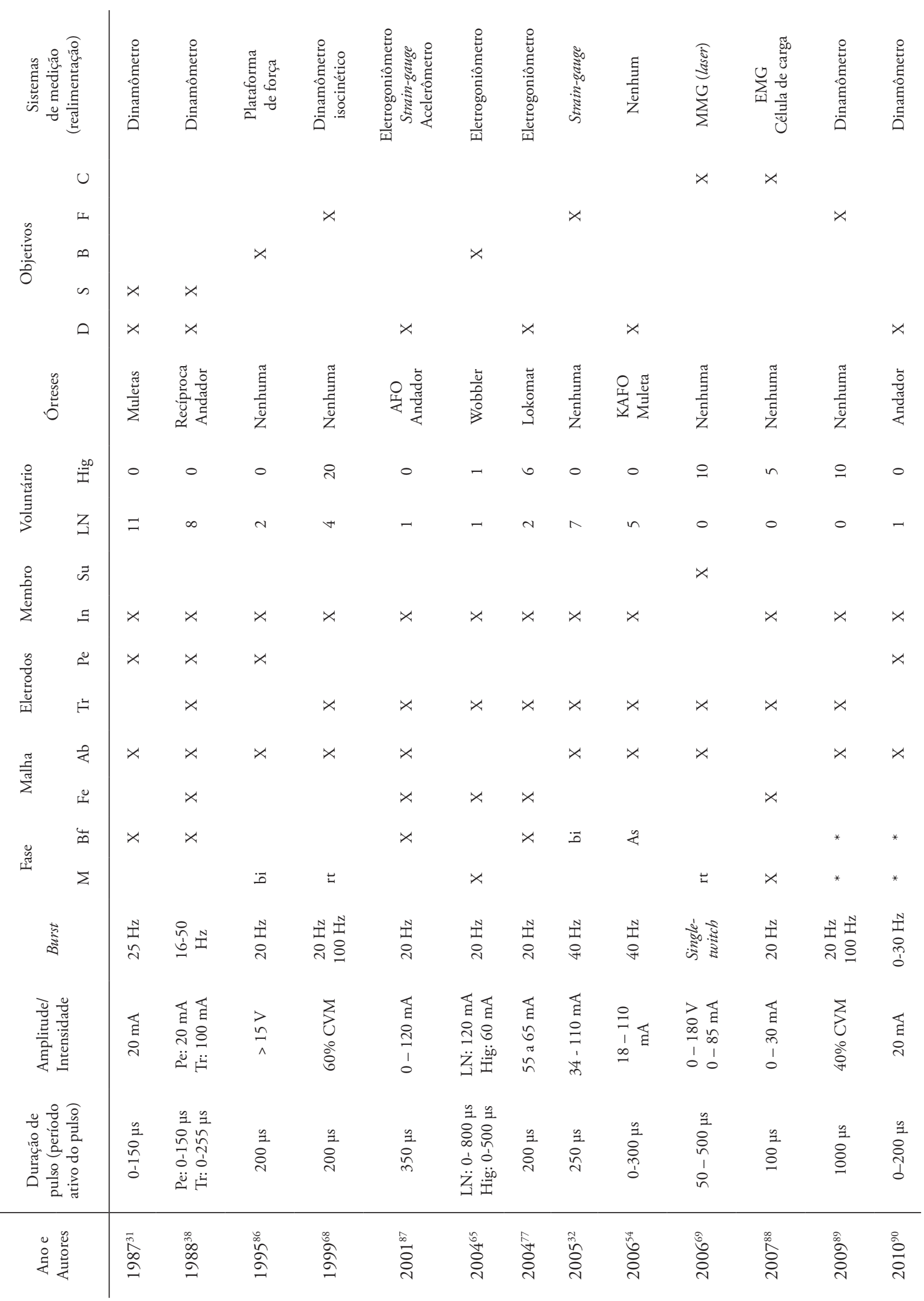

M-monofásica; Bf-bifásica; bi-bipolar; rt-retangular; as-assimétrica; Fe-fechada; Ab-aberta; Tr-transcutâneo; Pe-percutâneo; In-inferior; Su-superior; LN-lesão neurológica; Hig-hígido; AFO-órtese pé-tornozelo; KAFO-órtese pé-tornozelo-joelho; D-deambulação; S-subir escadas; B-bipedestação; F-fadiga muscular; C-contração muscular; ${ }^{*}$-não encontrado. 
pela flexão plantar e dorsiflexão do tornozelo. O estimulador elétrico utilizado era portátil com 32 canais de estimulação ${ }^{31}$. Passado um ano, 1988, melhorou-se o controle dos movimentos e agregaram ao sistema um estimulador elétrico transcutâneo ${ }^{38}$. O novo experimento foi realizado em malha aberta utilizando estimulação percutânea e transcutânea, concomitantemente. Oito participantes portadores de LM (T4 a T11) realizaram o movimento da marcha com passadas laterais e posteriores além de subir escadas com o auxílio de andador e/ou órtese de reciprocação. Os músculos que receberam eletrodos implantados foram o quadríceps femoral, iliopsoas, tensor da fáscia lata, grácil, sartório, tibial anterior, fibulares, gastrocnêmio, sóleo, semimembranáceo, glúteo máximo, porção posterior do adutor magno e glúteo médio. Os eletrodos de superfície foram posicionados nos músculos responsáveis pela extensão do tronco e quadrado do lombo. $\mathrm{O}$ intervalo entre os pulsos foi ajustado entre 20 e $60 \mathrm{~ms}$.

Trabalhou-se com FES percutânea em malha aberta para gerar o movimento de bipedestação com aplicação sobre o nervo motor, possibilitando o recrutamento de todas as fibras do músculo estimulado. Escolheram-se três pontos de estimulaçáo percutânea: (1) tronco e ramos do nervo femoral, para estimular todas as fibras do músculo quadríceps femoral; (2) tronco do nervo fibular comum, para atingir os músculos: tibial anterior, fibular curto, longo e anterior e (3) músculos glúteo máximo, glúteo médio e isquiotibiais. No programa de bipedestação, utilizaram-se apenas barras paralelas e nenhum sistema de medição de realimentação, pois se tratava de um estudo em malha aberta que propunha estimulação elétrica eficaz para manter a posição ortostática sem a necessidade do uso de órteses e com menos de $4 \%$ da massa corporal suprida como apoio ${ }^{86}$.

Pesquisadores tinham o objetivo de identificar qual frequência de burst, baixa $(20 \mathrm{~Hz})$ ou alta $(100 \mathrm{~Hz})$, promovia maior fadiga muscular. $\mathrm{O}$ protocolo consistiu de aplicaçóes da FES nas frequências baixa e alta para a realização de uma contração isométrica a $45^{\circ}$ de extensão máxima da articulação do joelho mensurada por meio de um dinamômetro isocinético adaptado ao sistema. Observou-se que a fadiga muscular foi maior para as aplicações em baixa frequência, em comparação com a alta, considerando o mesmo torque. Isso sugere que a estimu- lação ininterrupta em frequência $100 \mathrm{~Hz}$ aparenta ser melhor, quando se tem a intenção de manter contraçóes isométricas por um período de tempo maior ${ }^{68}$. Outros autores encontraram resultados similares em contraçóes concêntricas e excêntricas máximas e submáximas para voluntários hígidos com $\mathrm{FES}^{89}$.

Com o passar do tempo, uma série de pesquisas contribuiu para a evoluçâo tecnológica da aplicação da FES o que, consequentemente, trouxe melhoras aos portadores de LM; por exemplo, alteraçóes dos parâmetros da FES e inserção de novos sistemas de medição para realimentação e consequente controle. Foi desenvolvido um estimulador elétrico bipolar de 16 canais que podia ser usado tanto em malha aberta quanto fechada ${ }^{87}$. Utilizando órtese de tornozelo-pé (AFO) e um andador, obtiveram a deambulação de paciente com LM ao nível T9/T10. Estimulou-se o nervo fibular comum, o nervo femoral e os nervos glúteo superior e inferior. Os sistemas de medição utilizados para realimentação foram goniômetros analógicos posicionados lateralmente à articulação do joelho e da pelve, acelerômetros digitais na articulação da pelve e sensores analógicos de força na região plantar.

Avaliou-se o equilíbrio corporal, em um paciente hígido e outro com LM, utilizando FES híbrida para posição ortostática com as mãos livres. Um eletrogoniômetro foi utilizado na articulação do tornozelo para fornecer realimentação das alteraçóes angulares. Uma órtese denominada "Wobbler" foi confeccionada para manter a posição ortostática, fixando o tronco e mantendo os MMII em extensão. Aplicou-se também a FES no grupo muscular responsável pela flexão plantar de paciente com LM. Realizou-se um treino para fortalecimento muscular por doze semanas com FES no nervo tibial (músculo gastrocnêmio) e no nervo fibular profundo (músculo tibial anterior). Uma leve oscilação da postura era provocada por meio de um toque para frente e outro para trás, desestabilizando o equilíbrio do voluntário. A variação da angulação era percebida pelo sistema de realimentação que rapidamente a corrigia com o aumento ou reduçáo da duração do pulso estimulatório ${ }^{65}$.

Utilizou-se FES e uma órtese com eletrogoniômetros acoplados, denominada "Lokomat". A órtese mensurava a amplitude de movimento das articulaçóes do joelho e do quadril. O tipo de controle era de malha fechada 
e, por meio de processamento e modelagem matemática, controlava um sistema de servo-controle garantindo estabilidade ao movimento gerado pela FES. A FES foi aplicada em seis pacientes hígidos e dois com LM utilizando eletrodos de superfície sobre o quadríceps femoral. Durante $20 \mathrm{~s}$, a FES era aplicada com um período de descanso de $1 \mathrm{~min}$ para evitar a fadiga muscular ${ }^{77}$. Em estudo de caso com portador de LM, tendo sido o estimulador elétrico e os eletrodos implantados nos MMII, corroborou-se com a melhora da velocidade e coordenação da marcha após 36 sessóes ${ }^{90}$.

Avaliou-se a fadiga muscular em pacientes com LM. Para o grupo escolhido, dos três parâmetros estimulatórios (frequência, amplitude e duração de pulso), fixavam-se dois parâmetros, enquanto o terceiro variava $\pm 15 \%$ em relação ao seu valor médio, randomicamente. A alteração ocorria a cada $100 \mathrm{~ms}$. O torque foi medido por meio de uma célula de carga construída com strain-ganges. $\mathrm{O}$ critério estabelecido para a finalização do protocolo de fadiga foi o decaimento do torque em $70 \%$ do valor máximo obtido previamente ao protocolo. Os autores concluíram que a modulação randômica de frequência, duração do pulso e amplitude não produziu efeito sobre a fadiga muscular em contração isométrica para os músculos do quadríceps e tibial anterior, e que períodos de descanso de 10 min entre aplicações de FES eram ineficazes na recuperação das fibras musculares envolvidas no protocolo ${ }^{32}$. Em outro projeto, utilizou-se um sistema híbrido para deambulação com duas bengalas e órtese KAFO para um sistema em malha aberta, onde o disparo da FES era ativado por um contato mecânico (pushbutton). Os voluntários avaliados possuíam LM incompleta, com a capacidade de deambular com auxílio de muletas. Os músculos estimulados foram o glúteo máximo, quadríceps femoral, isquiotibiais, tibial anterior, gastrocnêmio e sóleo. Durante aproximadamente 10 semanas de treino, evidenciou-se o aumento da velocidade e largura da passada, comprovando o uso da FES como treino reabilitacional ${ }^{54}$.

Em recente estudo longitudinal com dois anos de duração com 20 voluntários com LM, avaliaram-se as transformaçóes corporais com técnicas de biópsia e tomografia computadorizada. Ao final do estudo, obtiveram (1) aumento das miofibrilas, (2) aumento de 75\% do diâmetro da coxa, (3) com 1187\% de aumento do torque muscular durante a FES e (4) $20 \%$ dos voluntários ao final do estudo conseguiam manter-se em posição ortostática com auxílio de barras paralelas e FES ${ }^{91}$.

Foi realizada uma estimulação híbrida com FES transcutânea e também contração voluntária de quatro voluntários hígidos em um sistema de malha fechada, onde os sinais de realimentação provinham do torque e da EMG do músculo tibial anterior. A intensidade da FES variava automaticamente mantendo o torque acima dos 30\% da contração voluntária máxima, sem a necessidade da intervenção do voluntário, mostrando a viabilidade do sistema em malha fechada ${ }^{88}$.

As frequências mais observadas nos protocolos que visam à movimentação muscular (e não fadiga) foram entre $20 \mathrm{~Hz}$ a $100 \mathrm{~Hz}$ (Tabela 1). Sendo que os eletrodos utilizados, na sua maioria foram transcutâneos. O período ativo do pulso na modulação PAM variou de $100 \mu \mathrm{s}$ até $500 \mu$ s. O uso de FES PAM ou PWM não demonstrou diferença na contração muscular, sendo bem dividido o seu uso pelos autores, o mesmo vale para o formato e tipo de onda escolhido. Os principais estudos que envolveram movimentos dinâmicos podem ser resumidos em trabalhos de fortalecimento e treinamento muscular, marcha, equilíbrio e compreensão, e estratégias para evitar a ocorrência de fadiga muscular. Os mecanismos de controle apresentaram-se mais eficazes quando utilizaram um sistema de malha fechada. Para a obtenção do biofeedback, diversos sensores de medição foram utilizados como célula de carga, EMG, MMG e eletrogoniômetro. As principais limitaçóes para uma prótese neural são as alteraçôes do padrão muscular ao longo do tempo como a fadiga, ou a capacidade do sistema de medição discernir essas alteraçóes fisiológicas e corrigi-las. Assim, novos estudos com o foco em padróes de FES e aprimoramento dos sistemas de medição poderão trazer novas perspectivas para a realização dessas próteses.

\section{CONCLUSÃO}

O homem busca mecanismos para suprir necessidades fisiológicas perdidas como o natural ato do movimento, o qual requer o circuito de ativação (SNC), sistema efetor (neuromuscular), o circuito sensorial (vias nervosas e neuronais ascendentes) realimentando o SNC, 
para que o evento continue se adaptando às alteraçóes proporcionadas pelos meios externo e interno. A aplicação de FES gera efeitos momentâneos como a movimentação articular por meio da contração muscular. Em longo prazo, a FES promove o fortalecimento do tecido muscular, melhora a condição cardiorrespiratória e desenvolve a plasticidade das vias neuronais. Sistemas que podem corrigir ou antecipar o processo da FES, sem que ocorra fadiga ou decaimento do torque motor, incrementam todos os benefícios da FES citados. Os resultados de parâmetros encontrados e de protocolos utilizados em pesquisas com FES são muito amplos. Com a evolução tecnológica, são desenvolvidos meios de controle artificial para o corpo humano, cujo objetivo é melhorar a qualidade de vida de pessoas que sofreram um dano físico parcial ou total. A FES, utilizada concomitantemente com sistemas de medição para ampliar sua aplicação, demarca os sistemas em malha fechada como de maior viabilidade reabilitacional para o homem quando acometido por patologias incapacitantes, como é o caso da LM. Uma perspectiva futura para a utilização da FES em malha fechada é o seu uso permanente como uma prótese neural. Estratégias de controle com FES poderiam, em tese, manter o paciente com o nível desejado de contração muscular, evitando alterações indesejáveis como adaptação do estímulo elétrico e/ou fadiga muscular. Trabalhos envolvendo o tema de FES em malha fechada são desenvolvidos por universidades e centros de pesquisa no exterior (Servia, Canadá, Japão, EUA) e no território brasileiro como, por exemplo, na UTFPR, PUCPR, USP, UNICAMP e UFSCAR.

\section{AGRADECIMENTOS}

Os autores agradecem à CAPES, à SETI-PR e ao CNPQ pelo suporte financeiro para a realização deste trabalho.

\section{REFERÊNCIAS}

1.Fodstad H, Hariz M. Electricity in the treatment of nervous system disease. Acta Neurochir Suppl 2007;97:11.

http://dx.doi.org/10.1007/978-3-211-33079-1_2
2.Popovic MR, Thrasher TA. Neuroprostheses. In: Bowlin GL, Wnek G (eds). Encyclopedia of Biomaterials and Biomedical Engineering. New York: Informa Healthcare, 2004, p.1056-65.

3.Galvani L. De viribus electricitatis in motu musculari commentarius. Bon Sci Art Inst Acad Comm 1791:363-418.

4.Verkhratsky A, Krishtal OA, Petersen OH. From Galvani to patch clamp: the development of electrophysiology. Pflugers Arch - Eur J Physiol 2006;453:233-47. 5.Duchenne GB. De l'Electrisation Localisee et de son Application a la Pathologie et a la Therapeutique. Paris: J-B Baillière, 1855, 900p.

6.DiLorenzo DJ, Bronzino JD. Neuroengineering. Boca Raton: CRC Press, 2007, 408p.

http://dx.doi.org/10.1201/9780849381850

7.He B. Neural engineering. Dordrecht: Kluwer/Plenum, 2005, 488p.

http://dx.doi.org/10.1007/b112182

8.Deluze C, Bosia L, Zirbs A, Chantraine A, Vischer TL. Electroacupuncture in fibromyalgia: results of a controlled trial. Br Med J 1992;305:1249-52.

http://dx.doi.org/10.1136/bmj.305.6864.1249

9.Song JW, Yang LJ, Russell SM. Peripheral nerve: what's new in basic science laboratories. Neurosurg Clin N Am 2009;20:121-31.

http://dx.doi.org/10.1016/j.nec.2008.07.026

10.Popovic MR, Curt A, Keller T, Dietz V. Functional electrical stimulation for grasping and walking: indications and limitations. Spinal Cord 2001;39:403-12. http://dx.doi.org/10.1038/sj.sc.3101191

11.O’Donovan KJ, O’Keeffe DT. Movement monitoring FES system. Annual Conference of the International Functional Electrical Stimulation Society, Cleveland, 2001, p. 1-3.

12.Graupe D, Kohn KH. Functional electrical stimulation for ambulation by paraplegics: Twelve years of clinical observations and system studies. Malabar: Krieger Publishing Company, 1994, 194p.

13.Isakov E, Mizrahi J, Najenson T. Biomechanical and physiological evaluation of FES-activated paraplegic patients. J Rehabil Res Dev 1986;23:9-19.

14.McNeil CJ, Murray BJ, Rice CL. Differential changes in muscle oxygenation between voluntary and stimulated isometric fatigue of human dorsiflexors. J Appl Physiol 2006;100:890-5.

http://dx.doi.org/10.1152/japplphysiol.00921.2005

15.Hamada T, Hayashi T, Kimura T, Nakao K, Moritani T. Electrical stimulation of human lower extremities enhances energy consumption, carbohydrate oxidation, and whole body glucose uptake. J Appl Physiol 2004;96:911-6. http://dx.doi.org/10.1152/japplphysiol.00664.2003

16.Packman-Braun R. Relationship between functional electrical stimulation duty cycle and fatigue in wrist extensor muscles of patients with hemiparesis. Phys Ther 1988;68:51-6.

17.Thorsen R, Spadone R, Ferrarin M. A pilot study of myoelectrically controlled FES of upper extremity. IEEE Trans Neural Syst Rehabil Eng 2001;9:161-8. http://dx.doi.org/10.1109/7333.928576

18. Hoshimiya N, Naito A, Yajima M, Handa Y. A multichannel FES system for the restoration of motor functions in high spinal cord injury patients: a respiration-controlled system for multijoint upper extremity. IEEE Trans Biomed Eng 1989;36:754-60.

http://dx.doi.org/10.1109/10.32108

19.Crago PE, Memberg WD, Usey MK, Keith MW, Kirsch RF, Chapman GJ, et al. An elbow extension neuroprosthesis for individuals with tetraplegia. IEEE Trans Rehabil Eng 1998;6:1-6.

http://dx.doi.org/10.1109/86.662614

20.Kilgore KL, Hart RL, Montague FW, Bryden AM, Keith MW, Hoyen HA, et al. An implanted myoelectrically-controlled neuroprosthesis for upper extremity function in spinal cord injury. Conf Proc IEEE Eng Med Biol Soc 2006; 1:1630-3. http://dx.doi.org/10.1109/IEMBS.2006.259939 
21.Maynard FM, Bracken MB, Creasey G, Ditunno JF, Donovan WH, Ducker $\mathrm{TB}$, et al. International standards for neurological and functional classification of spinal cord injury. Spinal Cord 1997;35:266-74.

http://dx.doi.org/10.1038/sj.sc.3100432

22.Burt AA. The epidemiology, natural history and prognosis of spinal cord injury. Curr Orthop 2004;18:26-32.

http://dx.doi.org/10.1016/j.cuor.2004.01.001

23.Lesão Medular: Principais Causas de Lesão Medular Traumática (Endereço na Internet). Brasília: SARAH - Rede Sarah Kubitschek de Hospitais. (atualizado em: 01/2010; acessado em: 01/2010). Disponível em: http://www.sarah. br/paginas/doencas/po/p_08_lesao_medular.htm.

24.Petrofsky JS. Electrical stimulation: neurophysiological basis and application. Basic Appl Myol 2004;14:205-13.

25.Venkatasubramanian G, Jung R, Sweeney JD. Functional Electrical Stimulation. In: Webster JG, ed. Encyclopedia of medical devices and instrumentation. 2 ed. New York, NY: John Wiley \& Sons, 2006, p.347-66.

26.Agne JE. Eletroterapia: Teoria e prática. Santa Maria: Orium, 2005, 336p. 27. Hatzis A, Stranjalis G, Megapanos C, Sdrolias PG, Panourias IG, Sakas DE. The current range of neuromodulatory devices and related technologies. Acta Neurochir Suppl 2007;97:21-9.

http://dx.doi.org/10.1007/978-3-211-33079-1_3

28.Ward AR, Shkuratova N. Russian electrical stimulation: the early experiments. Phys Ther 2002;82:1019.

29.Audu M, To C, Kobetic R, Triolo R. Gait evaluation of a novel hip constraint orthosis with implication for walking in paraplegia. IEEE Trans Neural Syst Rehabil Eng 2010; In Press.

http://dx.doi.org/10.1109/TNSRE.2010.2047594

30.To CS, Kirsch RF, Kobetic R, Triolo RJ. Simulation of a functional neuromuscular stimulation powered mechanical gait orthosis with coordinated joint locking. IEEE Trans Neural Syst Rehabil Eng 2005;13:227-35.

http://dx.doi.org/10.1109/TNSRE.2005.847384

31.Marsolais EB, Kobetic R. Functional electrical stimulation for walking in paraplegia. J Bone Joint Surg 1987;69:728-33.

32.Thrasher A, Graham GM, Popovic MR. Reducing muscle fatigue due to functional electrical stimulation using random modulation of stimulation parameters. Artif Organs 2005;29:453-8.

http://dx.doi.org/10.1111/j.1525-1594.2005.29076.x

33.Baker LL, Bowman BR, McNeal DR. Effects of waveform on comfort during neuromuscular electrical stimulation. Clin Orthop Relat Res 1988;233:75-85. 34.Rabischong E. Surface action potentials related to torque output in paraplegics' electrically stimulated quadriceps muscle. Med Eng Phys 1996;18:538-47. http://dx.doi.org/10.1016/1350-4533(96)00001-X

35.Rooney JG, Currier DP, Nitz AJ. Effect of variation in the burst and carrier frequency modes of neuromuscular electrical stimulation on pain perception of healthy subjects. Phys Ther 1992;72:800-6.

36.Geddes LA, Baker LE. Principles of applied biomedical instrumentation. 3 ed. New York: Wiley-Interscience, 1989, 961p.

37.Bronzino JD. Management of medical technology: a primer for clinical engineers. Boston: Butterworth-Heinemann, 1992, 451p.

38. Marsolais EB, Kobetic R. Development of a practical electrical stimulation system for restoring gait in the paralyzed patient. Clin Orthop Relat Res 1988;233:64-74.

39.Shimada Y, Sato K, Kagaya H, Konishi N, Miyamoto S, Matsunaga T. Clinical use of percutaneous intramuscular electrodes for functional electrical stimulation. Arch Phys Med Rehabil 1996;77:1014-8.

http://dx.doi.org/10.1016/S0003-9993(96)90061-1

40.Orizio C, Gobbo M, Diemont B. Changes of the force-frequency relationship in human tibialis anterior at fatigue. J Electromyogr Kinesiol 2004;14:523-30. http://dx.doi.org/10.1016/j.jelekin.2004.03.009

41.Castro MJ, Apple DF, Staron RS, Campos GER, Dudley GA. Influence of complete spinal cord injury on skeletal muscle within 6 mo of injury. J Appl Physiol 1999;86:350-8.

42.Valenga MH, Jorge RF, dos Santos A, Schneider Jr B, Nohama P. Sistema de estimulação elétrica gatilhado por sinal respiratório. 210 Congresso Brasileiro de Engenharia Biomédica, Salvador, 2008, p. 495-8.

43.Bear MF, Connors BW, Paradiso MA. Neurociências: Desvendando o sistema nervoso. 2 ed. Porto Alegre: Artmed, 2002, 855p.

44.Machado ABM. Neuroanatomia Funcional 2ed. São Paulo: Atheneu, 2006, $363 \mathrm{p}$.

45.Kandel ER, Jessell TM, Schwartz JH. Principles of neural science. 3 ed. New York: Elsevier 1991, 1138p.

46.Tonet O, Marinelli M, Citi L, Rossini PM, Rossini L, Megali G, et al. Defining brain-machine interface applications by matching interface performance with device requirements. J Neurosci Methods 2008;167: 91-104. http://dx.doi.org/10.1016/j.jneumeth.2007.03.015

47.Kandel ER, Jessell TM, Schwartz JH. Principles of neural science. 3 ed. New York: Elsevier 1991, 1137p.

48.Graziano MSA. Feedback remapping and the cortical control of movement. In: Latash ML, Lestienne F (eds). Motor control and learning. New York: Springer 2006:97-104.

http://dx.doi.org/10.1007/0-387-28287-4_9

49.Knikou M, Conway BA. Effects of electrically induced muscle contraction on flexion reflex in human spinal cord injury. Spinal Cord 2005;43:640-8. http://dx.doi.org/10.1038/sj.sc.3101772

50.Pierrot-Deseilligny E, Burke DC. The circuitry of the human spinal cord: its role in motor control and movement disorders. Cambridge: Univ Pr, 2005, 642p.

http://dx.doi.org/10.1017/CBO9780511545047

51.Loeb GE. Learning from the spinal cord. J Physiol 2001;533:111-7.

http://dx.doi.org/10.1111/j.1469-7793.2001.0111b.x

52.Kern H, Stramare R, Martino L, Gargiulo P, Carraro U. Permanent LMN denervation of human skeletal muscle and recovery by hb FES: management and monitoring. Eur J Translat Myol 2010;20:91-104.

53.Dietz V, Harkema SJ. Locomotor activity in spinal cord-injured persons. J Appl Physiol 2004;96:1954-60.

http://dx.doi.org/10.1152/japplphysiol.00942.2003

54.Thrasher TA, Flett HM, Popovic MR. Gait training regimen for incomplete spinal cord injury using functional electrical stimulation. Spinal Cord 2006;44:357-61.

http://dx.doi.org/10.1038/sj.sc.3101864

55.Vanderthommen M, Duchateau J. Electrical stimulation as a modality to improve performance of the neuromuscular system. Exerc Sport Sci Rev 2007;35:180-5.

http://dx.doi.org/10.1097/jes.0b013e318156e785

56.Higbie EJ, Cureton KJ, Warren Iii GL, Prior BM. Effects of concentric and eccentric training on muscle strength, cross-sectional area, and neural activation. J Appl Physiol 1996;81:2173-81.

57.Butler DS. Mobilização do sistema nervoso. Barueri: Manole, 2003, 270p. 58.Rushton DN. Functional electrical stimulation and rehabilitation - an hypothesis. Med Eng Phys 2003;25:75-8.

http://dx.doi.org/10.1016/S1350-4533(02)00040-1

59.Sheffler LR, Chae J. Neuromuscular electrical stimulation in neurorehabilitation. Muscle Nerve 2007;35:562-90.

http://dx.doi.org/10.1002/mus.20758

60.Lippold OCJ, Nicholls JG, Redfearn JWT. Electrical and mechanical factors in the adaptation of a mammalian muscle spindle. J Physiol 1960;153:209-17. 
61.Lieber RL, Kelly MJ. Torque history of electrically stimulated human quadriceps: implications for stimulation therapy. J Orthop Res 1993;11:131-41.

http://dx.doi.org/10.1002/jor.1100110115

62.Rushton DN. Functional electrical stimulation. Physiol Meas 1997;18:241-76. http://dx.doi.org/10.1088/0967-3334/18/4/001

63.Dietz V, Nakazawa K, Wirz M, Erni T. Level of spinal cord lesion determines locomotor activity in spinal man. Exp Brain Res 1999;128:405-9.

http://dx.doi.org/10.1007/s002210050861

64.Donaldson N, Yu CH. A strategy used by paraplegics to stand up using FES. IEEE Trans Rehabil Eng 1998;6:162-7.

http://dx.doi.org/10.1109/86.681181

65.Gollee H, Hunt KJ, Wood DE. New results in feedback control of unsupported standing in paraplegia. IEEE Trans Neural Syst Rehabil Eng 2004;12:73-80.

http://dx.doi.org/10.1109/TNSRE.2003.822765

66.Rueterbories J, Spaich EG, Larsen B, Andersen OK. Methods for gait event detection and analysis in ambulatory systems. Med Eng Phys 2010;32:545-52. http://dx.doi.org/10.1016/j.medengphy.2010.03.007

67.Bachschmidt RA, Harris GF, Simoneau GG. Walker-assisted gait in rehabilitation: a study of biomechanics andinstrumentation. IEEE Trans Neural Syst Rehabil Eng 2001; 9:96-105.

\section{http://dx.doi.org/10.1109/7333.918282}

68. Matsunaga T, Shimada Y, Sato K. Muscle fatigue from intermittent stimulation with low and high frequency electrical pulses. Arch Phys Med Rehabil 1999;80:48-53.

http://dx.doi.org/10.1016/S0003-9993(99)90306-4

69.McAndrew DJ, Rosser NAD, Brown JMM. Mechanomyographic measures of muscle contractile properties are influenced by the duration of the stimulatory pulse. J Appl Res 2006;6:142-52.

70.Krueger E, Scheeren E, Chu GFD, Nogueira-Neto GN, Button VLdSN. Mechanomyography analysis with $0.2 \mathrm{~s}$ and $1.0 \mathrm{~s}$ time delay after onset of contraction. BIOSTEC 2010: 3rd International Joint Conference on Biomedical Engineering Systems and Technologies, Valência, 2010, p.296-9.

71.Zhang Y, Frank CB, Rangayyan RM, Bell GD. Relationships of the vibromyogram to the surface electromyogram of the human rectus femoris muscle during voluntary isometric contraction. J Rehabil Res Dev 1996;33:395-403. 72.Nogueira-Neto GN, Müller RW, Salles FA, Nohama P, Button VLS. Mechanomyographic sensor: a triaxial accelerometry approach. International Joint Conference on Biomedical Engineering Systems and Technology, Funchal, 2008, p.176-9.

73.Seki K, Ogura T, Sato M, Ichie M. Changes of the evoked mechanomyogram during electrical stimulation. Annual Conference of the International Functional Electrical Stimulation Society, Brisbane, 2003.

74. Orizio C, Diemont B, Esposito F, Alfonsi E, Parrinello G, Moglia A, et al. Surface mechanomyogram reflects the changes in the mechanical properties of muscle at fatigue. Eur J Appl Physiol 1999;80:276-84.

http://dx.doi.org/10.1007/s004210050593

75.Krueger-Beck E, Scheeren E, Nogueira-Neto GN, Button VLdSN, Nohama P. Mechanomyographic Response during FES in Healthy and Paraplegic Subjects. 32nd Annual International Conference of the IEEE EMBS; Buenos Aires, 2010, p.626-9.

76.Krueger-Beck E, Scheeren E, Nogueira-Neto GN, Button VLdSN, Nohama P. Optimal FES Parameters Based on Mechanomyographic Efficiency Index. 32nd Annual International Conference of the IEEE EMBS, Buenos Aires, 2010, p.1378-81.

77.Jezernik S, Wassink RGV, Keller T. Sliding mode closed-loop control of FES: controlling the shank movement. IEEE Trans Biomed Eng 2004;51:263-72. http://dx.doi.org/10.1109/TBME.2003.820393

78.Kurosawa K, Futami R, Watanabe T, Hoshimiya N. Joint angle control by FES using a feedback error learning controller. IEEE Trans Neural Syst Rehabil Eng 2005; 13:359-71.

http://dx.doi.org/10.1109/TNSRE.2005.847355

79.Abbas JJ, Triolo RJ. Experimental evaluation of an adaptive feedforward controller foruse in functional neuromuscular stimulation systems. IEEE Trans Rehabil Eng 1997; 5:12-22.

http://dx.doi.org/10.1109/86.559345

80.Zhang D, Zhu K. Model and control of the locomotion of a biomimic musculoskeletal biped. Artif Life Robotics 2006;10:91-5.

http://dx.doi.org/10.1007/s10015-005-0369-1

81.Davoodi R, Andrews BJ. Fuzzy logic control of FES rowing exercise in paraplegia. IEEE Trans Biomed Eng 2004;51:541-3.

http://dx.doi.org/10.1109/TBME.2003.821043

82.Pai YC, Wening JD, Runtz EF, Iqbal K, Pavol MJ. Role of feedforward control of movement stability in reducing slip-related balance loss and falls among older adults. J Neurophysiol 2003; 90:755-62.

http://dx.doi.org/10.1152/jn.01118.2002

83.Patil PG, Carmena JM, Nicolelis MAL, Turner DA. Ensemble recordings of human subcortical neurons as a source of motor control signals for a brainmachine interface. Neurosurgery 2004;55:27-38.

84. Tonet O, Marinelli M, Citi L, Rossini PM, Rossini L, Megali G, et al. Defining brain-machine interface applications by matching interface performance with device requirements. J Neurosci Method 2008;167:91-104.

http://dx.doi.org/10.1016/j.jneumeth.2007.03.015

85.Taylor PN, Burridge JH, Dunkerley AL, Lamb A, Wood DE, Norton JA, et al. Patients' perceptions of the Odstock Dropped Foot Stimulator (ODFS). Clin Rehabil 1999; 13: 439-46.

http://dx.doi.org/10.1191/026921599677086409

86.Fujita K, Handa Y, Hoshimiya N, Ichie M. Stimulus adjustment protocol for FES-induced standing in paraplegiausing percutaneous intramuscular electrodes. IEEE Trans Rehabil Eng 1995;3:360-6.

http://dx.doi.org/10.1109/86.481976

87.Fisekovic N, Popovic DB. New controller for functional electrical stimulation systems. Med Eng Phys 2001;23:391-9.

http://dx.doi.org/10.1016/S1350-4533(01)00069-8

88.Langzam E, Nemirovsky Y, Isakov E, Mizrahi J. Muscle enhancement using closed-loop electrical stimulation: Volitional versus induced torque. J Electromyogr Kinesiol 2007;17:275-84.

http://dx.doi.org/10.1016/j.jelekin.2006.03.001

89.Baptista RR, Scheeren EM, Macintosh BR, Vaz MA. Low-frequency fatigue at maximal and submaximal muscle contractions. Braz J Med Biol Res 2009; 42:380-5.

http://dx.doi.org/10.1590/S0100-879X2009000400011

90.Bailey SN, Hardin EC, Kobetic R, Boggs LM, Pinault G, Triolo RJ. Neurotherapeutic and neuroprosthetic effects of implanted functional electrical stimulation for ambulation after incomplete spinal cord injury. J Rehab Res Develop 2010;47:7-16.

http://dx.doi.org/10.1682/JRRD.2009.03.0034

91.Kern H, Carraro U, Adami N, Biral D, Hofer C, Forstner C, et al. HomeBased Functional Electrical Stimulation Rescues Permanently Denervated Muscles in Paraplegic Patients With Complete Lower Motor Neuron Lesion. Neurorehabil Neural Repair 2010; In Press.

http://dx.doi.org/10.1177/1545968310366129 